\title{
On the Odd-Even Staggering of Mean Square Charge Radii in the Light Krypton and Strontium Region
}

\author{
P. LIEVENS 1,2 \\ PPE Division, CERN, CH-1211 Genève 23, Switzerland
}

E. ARnOlD ${ }^{3}$, W. BorChers ${ }^{4}$, U. GeORG, M. KeIM, A. KLeIn And R. NeUGART

Institut für Physik, Universität Mainz, D-55099 Mainz, Germany

L. VERMEEREN ${ }^{2}$

Instituut voor Kern- en Stralingsfysika, K.U. Leuven, B-3001 Leuven, Belgium

R.E. SILVERANS

Laboratorium voor Vaste-Stoffysika en Magnetisme, K.U. Leuven, B-3001 Leuven, Belgium

\begin{abstract}
Recently isotope shifts of ${ }^{72,74-96} \mathrm{Kr}$ and ${ }^{77-100} \mathrm{Sr}$ have been measured at the ISOLDE/ CERN mass separator facility by collinear laser spectroscopy. The deduced changes in mean square charge radii reveal sharp transitions in nuclear shape from spherical near the magic neutron number $\mathrm{N}=50$ towards strongly deformed for both the neutron deficient and neutron rich isotopes far from stability. The mean square charge radii of the neutron deficient isotopes exhibit a sign change of the odd-even staggering (OES), i.e. below the neutron number $\mathrm{N}=46$ the radius is systematically larger for the odd-N nuclei than for their even-N neighbours. This is in contrast to the situation of normal OES which is observed for the heavier isotopes. The inversion of the OES is interpreted as an effect of polarization, triggered by the addition of an unpaired neutron and driving the soft eveneven core into stable strong deformation.
\end{abstract}

accepted for publication in Europhysics Letters

(IS80 and IS304)

PACS. 21.10.Ft Charge distribution

PACS. 27.50.+e $59 \leq \mathrm{A} \leq 89$

PACS. 27.60.+j $90 \leq \mathrm{A} \leq 149$

1 Present address: Laboratorium voor Vaste-Stoffysika en Magnetisme, K.U. Leuven, B-3001 Leuven, Belgium

2 Senior Research Assistant of the Belgian National Fund for Scientific Research (N.F.W.O.)

3 Present address: Heraeus Noblelight GmbH, Hanau, Germany

4 Present address: Agfa Gevaert AG, Leverkusen, Germany 
Nuclear mean square charge radii $\left(\left\langle\mathrm{r}^{2}\right\rangle\right)$ give precise information about the nuclear size, but they also reflect properties such as the shape and certain surface effects of the nuclear ground state. A unique access to radii of unstable nuclei is provided by the measurement of optical isotope shifts [1]. From these, the changes in mean square (ms) charge radii within a sequence of isotopes are obtained by applying generally well known atomic calibration factors, in some cases combined with muonic isotope shift data [2]. This information can be complemented by absolute values of the radii of stable isotopes (obtained, e.g., from electron scattering experiments [3]). However, valuable physics information is also given by the one-by-one changes in $\mathrm{ms}$ charge radii between isotopes with neighbouring mass numbers $A$ and $\left.A^{\prime}\left(\delta<r^{2}\right\rangle A, A^{\prime}=\left\langle r^{2}\right\rangle A^{\prime}-\left\langle r^{2}\right\rangle A\right)$. This differential behaviour is rather independent of the models used to calibrate the absolute values of $\left\langle\mathrm{r}^{2}\right\rangle$ or of $\delta<\mathrm{r}^{2}>\mathrm{A}, \mathrm{A}^{\prime}$ for large differences between $\mathrm{A}$ and $\mathrm{A}^{\prime}$.

A particular property of radii is the so-called odd-even staggering (OES). This is the systematic difference of the radii of odd-N nuclei with respect to their even-N neighbours. Although this OES is very small in comparison to the total radius, it is given directly by isotope shift measurements of $\delta<\mathrm{r}^{2}>\mathrm{A}, \mathrm{A}^{\prime}$.

The region of light krypton and strontium nuclei is particularly interesting for detailed studies of ground state properties. Theoretical predictions and experimental data show a competition between completely different shapes to be energetically most favourable [4]. Some nuclei (e.g. ${ }^{76,77,79} \mathrm{Sr},{ }^{74} \mathrm{Kr}$ ) are known to be nearly perfect rigid rotors with ground state deformations among the largest known, while others (e.g. ${ }^{80,82} \mathrm{Sr},{ }^{76,78} \mathrm{Kr}$ ) clearly exhibit vibrational properties. It is evident that rapid shape transitions and shape instabilities occur when the nuclei approach the $\mathrm{N}=\mathrm{Z}$ line.

Present techniques of laser spectroscopy are sensitive enough to make isotope shift measurements possible over a large range of neutron numbers including isotopes far from stability of which only small quantities can be produced. The measurements on the series of $\mathrm{Kr}$ and $\mathrm{Sr}$ isotopes discussed here, were carried out by collinear laser spectroscopy [5], with the implementation of extremely sensitive ion or atom counting techniques. These are based on optical pumping and state selective charge-changing collisions [6]. The experiments are treated in detail in ref. [7-11] and they are complemented by an experiment on the odd- $\mathrm{Z}$ isotopic series of $\mathrm{Rb}[12]$.

In this Letter we will focus on the discussion of the OES of ms charge radii and the remarkable inversion observed for both the light $\mathrm{Kr}$ and $\mathrm{Sr}$ isotopes. This phenomenon is similar but less clearly developed in the $\mathrm{Rb}$ case.

The global changes in ms charge radii for $\mathrm{Kr}$ [11] and $\mathrm{Sr}$ [7-10] are presented in FIGURE 1 as a function of the neutron number N. An illustrative presentation of details is given in FIGURE 2, where the one-by-one changes of the radii $\left.\delta<\mathrm{r}^{2}\right\rangle \mathrm{N}-1, \mathrm{~N}$ are plotted versus $\mathrm{N}$ for the $\mathrm{Kr}$ and $\mathrm{Sr}$ ground and isomeric states. The odd-A isomers are evaluated with 
respect to their even-even neighbours in the ground state. The $\delta<\mathrm{r}^{2}>\mathrm{N}-1, \mathrm{~N}$ values are also listed in TABLE 1.

An important contribution to the ms charge radii stems from nuclear deformation. To first order this can be expressed by the droplet model formula [13]

$$
<r^{2}>^{N} \approx \frac{3}{5} R_{Z}^{2}(N)\left(1+\frac{5}{4 \pi}<\beta^{2}>^{N}\right)
$$

with $\mathrm{R}_{\mathrm{Z}}$ the proton sharp radius and $\left\langle\beta^{2}\right\rangle \mathrm{N}$ the ms quadrupole deformation. Isodeformation curves for $\delta<\mathrm{r}^{2}>\mathrm{N}^{\prime}=50, \mathrm{~N}$ using the droplet parameters given in ref. [14] are included in FIGURE 1.

Although this traditional interpretation of radii in the framework of a macroscopic model including deformation fails in the neighbourhood of shell closures (see $[7,8,11]$ ), it can serve as a guide to the understanding of unusual odd-even effects occurring in the region where the nuclei approach strongly deformed shapes. The OES is most transparently presented in terms of the difference $\mathrm{D}$ between the radius of an odd- $\mathrm{N}$ isotope and the mean value of the radii of its even- $\mathrm{N}$ neighbours,

$$
\begin{aligned}
D & =\left\langle r^{2}>^{N}-\frac{1}{2}\left(<r^{2}>^{N-1}+<r^{2}>^{N+1}\right)\right. \\
& =\delta<r^{2}>^{N-1, N}-\frac{1}{2} \delta<r^{2}>^{N-1, N+1} .
\end{aligned}
$$

The values of $\mathrm{D}$ as a function of $\mathrm{N}$ are plotted in FIGURE 3.

For historical reasons (because this is observed in most cases), a smaller radius for the odd-N isotope (i.e. $\mathrm{D}<0$ ) is called normal OES. Such an effect is found for the $\mathrm{Kr}$ and $\mathrm{Sr}$ ground states with masses between $\mathrm{N}=47$ and $\mathrm{N}=61$, and for the isomers down to ${ }^{83} \mathrm{Sr}$ and ${ }^{79} \mathrm{Kr}$.

Normal OES in near-spherical nuclei has been described by Reehal and Sörensen in a pairing-plus-quadrupole model [15]. Pairing leads to enlarged vibrational zero-point motion reflected in a higher $\left\langle\beta^{2}\right\rangle$ and larger $\left\langle\mathrm{r}^{2}\right\rangle$ for the even-N isotopes compared to their neighbours containing an unpaired neutron. A microscopic theoretical approach reproduces the OES by the inclusion of 3- or 4-body interactions in the effective force of Hartree-Fock calculations [16,17].

Rare cases of inverted OES have been traced to unusual situations in the nuclear structure. In the series ${ }^{221-225} \mathrm{Ra}$ the unpaired nucleon polarizes the even-even core towards pronounced octupole deformation [18]. Similar arguments were used to interpret the inverted OES in the Eu isotopes with $\mathrm{N}=89-92$ [19]. In other cases the inverted OES provided experimental evidence for shape coexistence. The huge staggering of radii in the light $\mathrm{Hg}$ region was explained by coexisting weak oblate and strong prolate deformation minima whose relative depth depends on the presence of an unpaired neutron [20]. 
The staggering of the $\mathrm{Kr}$ and $\mathrm{Sr}$ isotopes with $46 \leq \mathrm{N} \leq 58$ can be explained by the traditional description of normal OES. In the nuclei with $\mathrm{N}>50$, the proximity of the spherical subshell closure at $\mathrm{Z}=38$ combined with the filling of the $2 \mathrm{~d}_{5 / 2}$ neutron orbital $(50 \leq \mathrm{N} \leq 56)$ keeps a spherical even- $\mathrm{N}$ and odd- $\mathrm{N}$ shape. This is clearly seen in the energy spectra with a high-lying excited $0_{2}{ }^{+}$state, connected to the ground state $0_{1}{ }^{+}$ with a small $\rho^{2}(E 0)$ value [4]. No spatial preference directions nor polarization effects show up, resulting in a standard OES pattern. It is only for $\mathrm{Sr}$ beyond $\mathrm{N}=58$ that a large prolate deformation sets in as observed in the sudden increase of the ms radius and also in the related very large $\rho^{2}(\mathrm{E} 0)$ values. This corresponds to a pronounced odd-even effect occurring between $\mathrm{N}=59$ and 60 . The isotone ${ }^{96} \mathrm{Kr}$, on the contrary, remains quasi-spherical (see ref. [11]).

The situation changes below $\mathrm{N}=50$ where a more gradual change towards deformed shapes develops (as predicted by potential energy surface calculations [21-23]). Here the $\mathrm{Sr}$ and $\mathrm{Kr}$ radii exhibit a rather similar OES behaviour including the sign change of the parameter $\mathrm{D}$ at neutron number $\mathrm{N}=45$.

We ascribe this inversion (i.e. $\mathrm{D}<0$ for odd $\mathrm{N}$ ) and the pronounced increase of this effect towards ${ }^{75} \mathrm{Kr}(\mathrm{N}=39)$ to a polarization of the even-even core by the additional neutron. Experimentally, the rigid-rotor-like behaviour of the odd-A neutron deficient ${ }^{77} \mathrm{Sr},{ }^{79} \mathrm{Sr}$ and ${ }^{75} \mathrm{Kr},{ }^{77} \mathrm{Kr}$ is established through the studies of their high spin states [24-30]. The same conclusion can be drawn from the nuclear moments measured by laser spectroscopy $[8,10,11]$.

Also a different gross behaviour of the ms radii is observed for the even-A and odd-A isotopes: while the odd-A isotopes continue to develop stronger deformation, as reflected in the steady increase of $\delta<\mathrm{r}^{2}>50, \mathrm{~N}$ towards lower neutron number, the deformation of the even- $A$ isotopes saturates at $A=76$ and decreases towards $A=72$. Obviously the even-A isotopes remain somehow soft. This can be explained by the depletion of the $1 \mathrm{~g}_{9 / 2}$ neutron orbital, an orbital with a longer radial extent than the pfshell orbitals. Rather flat energy surfaces towards $\gamma$-soft develop near $\mathrm{N}=\mathrm{Z}$. It is precisely in this region that fluctuations in the nuclear radius become possible whenever a neutron is added to the even-even system, polarizing the total nucleus to prolate shapes by populating the orbitals from the down-sloping $1 \mathrm{~g}_{9 / 2}$ and $1 \mathrm{~h}_{11 / 2}(\mathrm{low}-\Omega$ ) states at $\varepsilon \approx$ 0.3-0.4. This situation is very reminiscent of the odd $\mathrm{Hg}$ nuclei where much stronger polarization effects have been observed.

Remarkable is also the different staggering behaviour of ground states and isomers with the same neutron configuration. This is illustrated by the $\mathrm{I}=7 / 2$ nuclei ${ }^{79} \mathrm{~m}, 81 \mathrm{Kr}$ and ${ }^{83} \mathrm{Sr}$ with the anomalously coupled $\left(\lg _{9 / 2}{ }^{-3}\right)_{7 / 2}$ neutron configuration. While the staggering for ${ }^{81} \mathrm{Kr}$ and ${ }^{83} \mathrm{Sr}$ is inverted, it is normal for ${ }^{79} \mathrm{~m} \mathrm{Kr}$. This is consistent with the smaller deformation of $79 \mathrm{~m} \mathrm{Kr}$ suggested by the smaller quadrupole moment [11]. For all 
isomeric states the OES remains normal, even below $\mathrm{N}=45$, regardless of the single particle state that is occupied. This clearly indicates that in the delicate balance between shape-coexistent states with different deformations, the strongly deformed states are energetically favoured.

The importance of core-polarization effects in this region has already been demonstrated by the results of high spin studies for ${ }^{81} \mathrm{Sr}$ [31]. Here several rotational bands are found, each built on a different single-particle configuration. The persistence of inverted OES towards lighter isotopes thus indicates that core polarization effects continue to be important for the $\mathrm{N}=45$ isotopes ${ }^{81} \mathrm{Kr}$ and ${ }^{83} \mathrm{Sr}$.

In conclusion, we state that measurements of the changes in ms charge radii for light $\mathrm{Kr}$ and $\mathrm{Sr}$ isotopes reveal direct information on the odd-even shape differences. The sign change of the odd-even staggering is seen as experimental evidence for the stabilization of large deformation of the soft even-even core by the addition of a neutron. It is our hope that this qualitative discussion will stimulate more quantitative theoretical work.

\section{ACKNOWLEDGMENTS}

We wish to thank K. Heyde and W. Nazarewicz for their valuable comments and suggestions. This work was supported by the German Bundesministerium für Forschung und Technologie (B.M.F.T.) under Contracts No. 06 MZ 188 I and 06 MZ 501 I, the Belgian National Fund for Scientific Research (N.F.W.O.) and the Interuniversitary Institute for Nuclear Sciences (I.I.K.W.). 


\section{REFERENCES}

[1] OtTEN E.-W., Treatise on Heavy Ion Physics, vol. 8: Nuclei far from Stability, ed. D.A. BROMLY, (Plenum, New York, 1988), p. 517.

[2] Engfer R. ET AL., At Data Nucl. Data Tables, 14 (1974) 509.

[3] EMrich H.J. ET AL., Nucl. Phys. A, 396 (1983) 401c.

[4] Proc. of the Int. Workshop on Nuclear Structure in the Zr Region, eds. Eberth J., MEYER R.A. AND SISTEMICH K., Bad Honnef, 1988 (Springer Verlag, Berlin, 1988).

[5] Neugart R., Progress in Atomic Spectroscopy, part D, eds. BeYER H.J. AND KLEINPOPPEN H. (Plenum, New York, 1987) p.75.

[6] LIEVENS P. ET AL., Nucl. Instr. Meth., B70 (1992) 532.

[7] Silverans R.E. ET Al., Phys. Rev. Lett., 60 (1988) 2607.

[8] BuChInger F. ET AL., Phys. Rev. C., 41 (1990) 2883.

[9] Lievens P. ET AL., Phys. Lett., B256 (1991) 141.

[10] LIEVEns P. ET AL., Phys. Rev. C, 46 (1992) 797.

[11] KeIM M. ET AL., Nucl. Phys. A, 586 (1995) 219.

[12] Thibault C. ET AL., Phys. Rev. C, 23 (1981) 2720.

[13] Myers W.D. AND SCHMIDT K.H., Nucl. Phys. A, 410 (1983) 61.

[14] MÖLler P. ET AL., At. Data Nucl. Data Tables, 39 (1988) 225.

[15] ReEHAl B.S. AND SÖRENSEN R.A., Nucl. Phys. A, 161 (1971) 385.

[16] Zawischa D., RegGe U. And Stapel R., Phys. Lett. B, 185 (1987) 299.

[17] RegGe U. And Zawischa D., Phys. Rev. Lett., 61 (1988) 149.

[18] Ahmad S.A. ET AL., Nucl. Phys. A, 483 (1988) 244.

[19] Alkhazov G.D. ET AL., Z. Phys. A - Atomic Nuclei, 337 (1990) 257.

[20] Ulm G. ET AL., Z. Phys. A - Atoms and Nuclei, 325 (1986) 247.

[21] Heyde K., Moreau J. And Waroquier M., Phys. Rev. C, 29 (1984) 1859.

[22] NAZAREWICZ W. ET AL., Nucl. Phys. A, 435 (1985) 397.

[23] BONCHE P. ET AL., Nucl. Phys. A, 443 (1985) 39.

[24] Lister C.J. ET AL., Phys. Rev. Lett., 49 (1982) 308.

[25] HeEse J. ET AL., Phys. Rev. C, 41 (1990) 603.

[26] Chishti A.A. ET AL., Nucl. Phys. A, 501 (1989) 568.

[27] Winchell D.F. ET AL., Phys. Rev. C, 40 (1989) 2672.

[28] Skoda S. ET AL., Z. Phys. A - Atomic Nuclei, 336 (1990) 391.

[29] Cardonna M.A. et al, Phys. Rev. C, 42 (1990) 591.

[30] Johnson T.D. ET AL., Phys. Rev. C, 42 (1990) 2418.

[31] Moore E.F. ET AL., Phys. Rev. C, 38 (1988) 696. 


\section{TABLE I}

Differential changes in mean square charge radii $\mathrm{d}<\mathrm{r}^{2}>\mathrm{N}-1, \mathrm{~N}\left(\mathrm{fm}^{2}\right)$ for the $\mathrm{Kr}$ and $\mathrm{Sr}$ ground and isomeric states. The quoted errors are purely statistical. A thorough discussion of the statistical and systematic errors and values for the systematic errors are given in refs. $[8,11]$.

\begin{tabular}{|c|c|c|c|c|}
\hline $\mathbf{N}$ & $\mathbf{K r}$ & $\mathrm{Sr}$ & $\mathbf{m}_{\mathbf{K r}}$ & $\mathrm{mSr}$ \\
\hline 39 & $0.191(9)$ & & & \\
\hline 40 & $-0.065(8)$ & $-0.006(14)$ & & \\
\hline 41 & $0.053(6)$ & $0.019(10)$ & & \\
\hline 42 & $-0.037(5)$ & $-0.018(9)$ & & \\
\hline 43 & $-0.004(5)$ & $-0.011(9)$ & $-0.046(5)$ & \\
\hline 44 & $-0.054(8)$ & $-0.053(8)$ & $-0.012(8)$ & \\
\hline 45 & $-0.015(8)$ & $-0.017(7)$ & $-0.034(11)$ & $-0.045(7)$ \\
\hline 46 & $-0.028(5)$ & $-0.046(5)$ & $-0.009(9)$ & $-0.018(5)$ \\
\hline 47 & $-0.040(4)$ & $-0.068(4)$ & $-0.034(7)$ & $-0.041(4)$ \\
\hline 48 & $0.011(3)$ & $0.002(4)$ & $0.005(6)$ & $-0.025(4)$ \\
\hline 49 & $-0.033(4)$ & $-0.043(3)$ & $-0.070(5)$ & $-0.074(4)$ \\
\hline 50 & $-0.009(4)$ & $-0.007(2)$ & $0.028(5)$ & $0.024(3)$ \\
\hline 51 & $0.125(3)$ & $0.124(1)$ & & \\
\hline 52 & $0.157(5)$ & $0.153(4)$ & & \\
\hline 53 & $0.097(6)$ & $0.097(5)$ & & \\
\hline 54 & $0.116(11)$ & $0.138(6)$ & & \\
\hline 55 & $0.102(12)$ & $0.078(6)$ & & \\
\hline 56 & $0.154(8)$ & $0.125(7)$ & & \\
\hline 57 & $0.060(6)$ & $0.087(8)$ & & \\
\hline 58 & $0.178(6)$ & $0.166(8)$ & & \\
\hline 59 & $0.056(5)$ & $0.079(9)$ & & \\
\hline 60 & $0.172(10)$ & $0.578(9)$ & & \\
\hline 61 & & $0.093(10)$ & & \\
\hline 62 & & $0.114(17)$ & & \\
\hline
\end{tabular}


FIGURE 1:

Figure 1

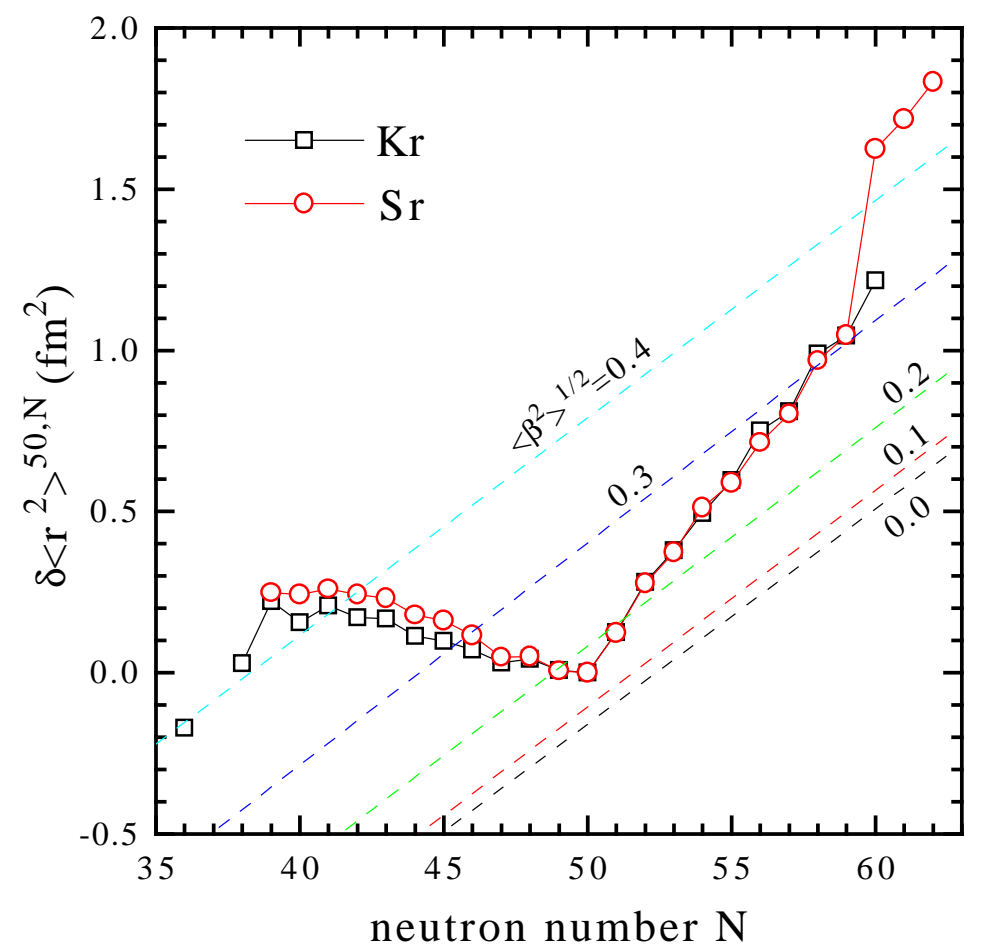

Changes in mean square charge radii for $\mathrm{Kr}$ and $\mathrm{Sr}$ isotopes versus neutron number. Droplet model isodeformation curves are included as dashed lines. 
FIGURE 2:

Figure 2

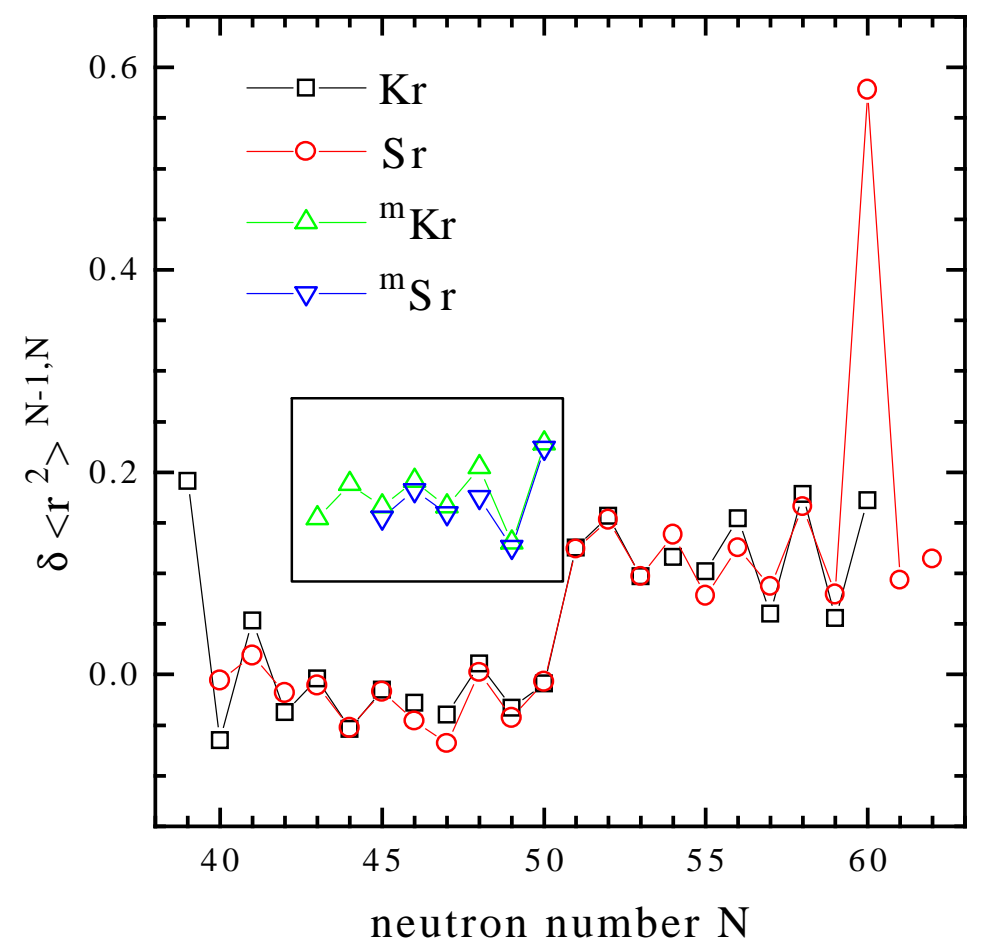

Differential changes in mean square charge radii for $\mathrm{Kr}$ and $\mathrm{Sr}$ ground and isomeric states. The values for the isomers (in the inset) are shifted by $0.2 \mathrm{fm}^{2}$. 
FIGURE 3:

Figure 3

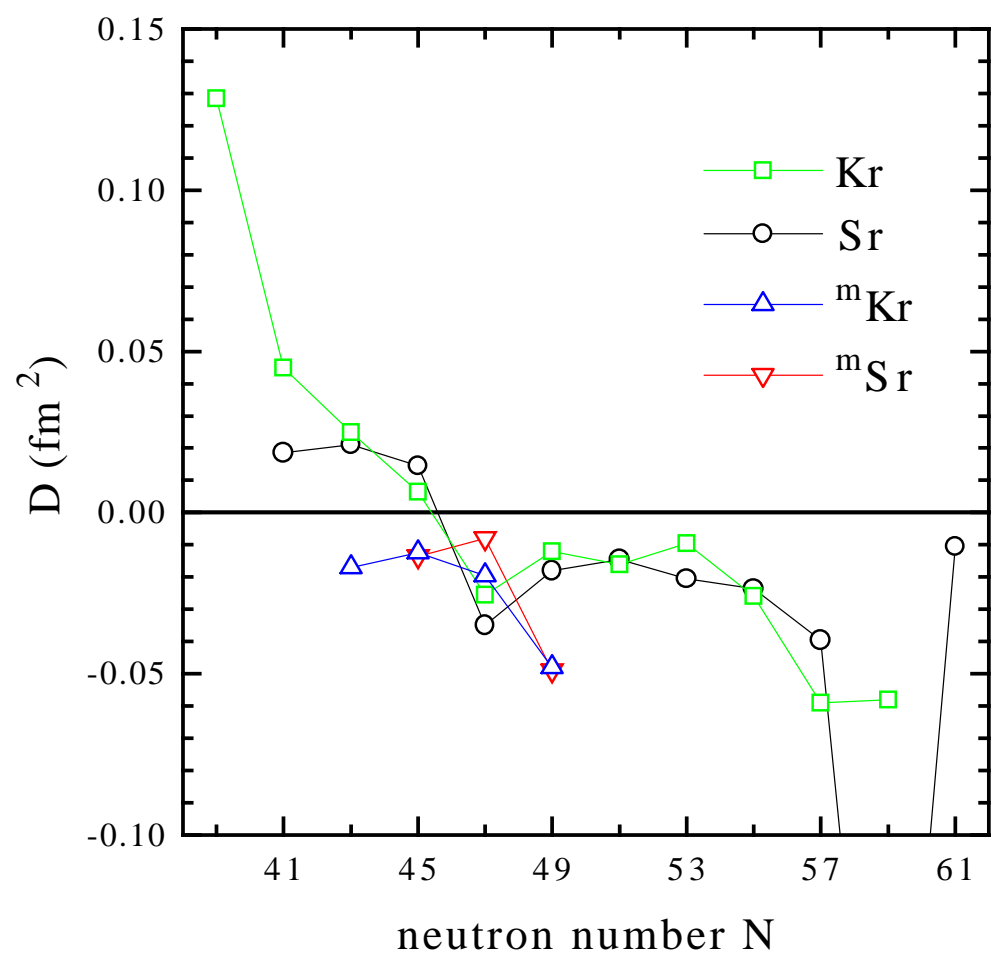

The odd-even staggering D defined in formula (2) for the odd- $\mathrm{N} \mathrm{Kr}$ and $\mathrm{Sr}$ isotopes. Values for the isomers are also included. 